\title{
ON THE ALMOST SPLIT SEQUENCES FOR RELATIVELY PROJECTIVE MODULES OVER A FINITE GROUP
}

\author{
MARK KLEINER
}

(Communicated by Maurice Auslander)

\begin{abstract}
Let $G$ be a finite group with a subgroup $H$. Given a field $k$ of characteristic $p$ dividing the order of $G$, denote by $\bmod k G$ the category of finite-dimensional over $k$ left $G$-modules, and let $\mathscr{C}$ be the full subcategory of mod $k G$ determined by the relatively projective modules. Let $0 \rightarrow L \rightarrow$ $M \rightarrow N \rightarrow 0$ be an exact sequence in $\bmod k G$ with $L, M, N \in \mathscr{C}$. It is proved that the sequence is an almost split sequence in $\mathscr{C}$ if and only if it is an almost split sequence in mod $k G$. This implies, together with a recent result of Carlson and Happel, that $\mathscr{C}$ has almost split sequences if and only if it is closed under extensions, i.e., if and only if $p$ is coprime to either the order of $H$ or the index of $H$ in $G$.
\end{abstract}

Throughout the paper, we fix an artin algebra $\Lambda$ [1] with Jacobson radical $\mathbf{r}$ and denote by $\bmod \Lambda$ the category of finitely generated left $\Lambda$-modules. We also fix $\mathscr{C}$, a full subcategory of $\bmod \Lambda$ closed under isomorphisms, direct sums, and direct summands. Denote by ind $\mathscr{C}$ the set of pairwise nonisomorphic indecomposable modules in $\mathscr{C}$, and write ind $\Lambda$ for $\operatorname{ind}(\bmod \Lambda)$. We say that $\mathscr{C}$ is closed under $D \operatorname{Tr}$ and $\operatorname{Tr} D$ if for each $C \in$ ind $\mathscr{C}, D \operatorname{Tr} C$ and $\operatorname{Tr} D C$ [2] are in $\mathscr{C}$. We freely use the notions of a functorially finite subcategory and of a left or right almost split morphism in $\mathscr{C}$, introduced in $[5,6]$ as a generalization of the corresponding notions in [2].

The only modification is that we replace Ext-projective and Ext-injective modules in $\mathscr{C}$ [5] by extension projective and extension injective modules, respectively, which are defined as follows. A module $N \in \mathscr{C}$ is called extension projective in $\mathscr{C}$ if every exact sequence $0 \rightarrow X \rightarrow Y \rightarrow N \rightarrow 0$ in $\mathscr{C}$ splits. Recall that a module $N \in \mathscr{C}$ is called Ext-projective in $\mathscr{C}$ if $\operatorname{Ext}_{\Lambda}^{1}(N, X)=0$ for all $X \in \mathscr{C}$. Clearly, every Ext-projective module is extension projective. On the other hand, in the context of [5], $\mathscr{C}$ is closed under extensions, i.e., in every exact sequence $0 \rightarrow X \rightarrow Y \rightarrow Z \rightarrow 0$ with $X, Z \in \mathscr{C}$, we have $Y \in \mathscr{C}$ so that a module is Ext-projective if and only if it is extension projective. Extension injective modules in $\mathscr{C}$ are introduced dually.

Received by the editors November 20, 1990 and, in revised form, April 22, 1991.

1980 Mathematics Subject Classification (1985 Revision). Primary 16A64, 16A46, 16A27.

Key words and phrases. Almost split sequence, relatively projective module, finite group.

The author was partially supported by NSA grant $89-\mathrm{H}-2032$. 
Recall that an exact sequence $0 \rightarrow L \stackrel{f}{\rightarrow} M \stackrel{g}{\rightarrow} N \rightarrow 0$ in $\mathscr{C}$ is called almost split in $\mathscr{C}$ if $f$ is a left almost split morphism in $\mathscr{C}$ and $g$ is a right almost split morphism in $\mathscr{C} . \mathscr{C}$ is said to have almost split sequences if it satisfies the following conditions:

(a) $\mathscr{C}$ has almost split morphisms.

(b) If $N \in$ ind $\mathscr{C}$ is not extension projective, then there is an almost split sequence $0 \rightarrow L \rightarrow M \rightarrow N \rightarrow 0$ in $\mathscr{C}$.

(c) Dual to (b).

It is obvious that if an exact sequence $0 \rightarrow L \rightarrow M \rightarrow N \rightarrow 0$ in $\mathscr{C}$ is almost split in $\bmod \Lambda$, then it is almost split in $\mathscr{C}$. The converse is true only under certain conditions, as was pointed out by K. W. Roggenkamp, whose short proof replaces here an argument of the author.

Propositon 1. (a) Let $0 \rightarrow L \stackrel{f}{\rightarrow} M \stackrel{g}{\rightarrow} N \rightarrow 0$ be an almost split sequence in $\mathscr{C}$, and let $0 \rightarrow U \stackrel{s}{\rightarrow} V \stackrel{t}{\rightarrow} N \rightarrow 0$ be the almost split sequence in $\bmod \Lambda$ with right end $N$, where $U \in \mathscr{C}$. Then the sequences are isomorphic.

(b) If $\mathscr{C}$ is closed under DTr and $\operatorname{TrD}$, then a short exact sequence in $\mathscr{C}$ is almost split in $\mathscr{C}$ if and only if it is almost split in $\bmod \Lambda$.

Proof. (a) Since $g$ is not a splittable epimorphism, we have the following exact commutative diagram:

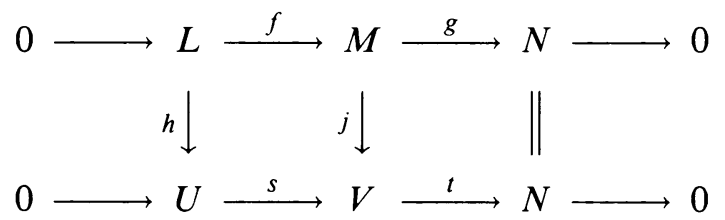

If $h$ is not a splittable monomorphism, it can be extended to $M$ because $U \in \mathscr{C}$ and $f$ is a left almost split morphism in $\mathscr{C}$. Then the bottom row splits, a contradiction. Thus, $h$ is a splittable monomorphism and must be an isomorphism because $U \in$ ind $\Lambda$.

(b) Follows from (a) and its dual.

Proposition 2. Let $\Lambda$ be connected. Suppose that

(i) $\mathscr{C}$ has almost split sequences and is closed under DTr and $\operatorname{TrD}$,

(ii) all extension projectives in $\mathscr{C}$ are projective in $\bmod \Lambda$,

(iii) all extension injectives in $\mathscr{C}$ are injective in $\bmod \Lambda$,

(iv) $\mathscr{C}$ is functorially finite.

Then either $\mathscr{C}=\bmod \Lambda$ or $\mathscr{C}$ consists of projective-injective modules in $\bmod \Lambda$; in particular, $\mathscr{C}$ is closed under extensions.

Proof. Let $\mathscr{C}$ satisfy (i) and (ii), and suppose $L \in$ ind $\mathscr{C}$ is not extension injective. Show that if $X \rightarrow L$ and $L \rightarrow Y$ are irreducible maps with $X, Y \in$ ind $\Lambda$, then $X, Y \in \mathscr{C}$. Really, by assumption, there is an almost split sequence $0 \rightarrow L \rightarrow M \rightarrow N \rightarrow 0$ in $\mathscr{C}$ that is almost split in mod $\Lambda$ by Proposition 1(b). Then $Y \in \mathscr{C}$ for $Y$ is a direct summand of $M$. If $L$ is not extension projective in $\mathscr{C}$, there is an almost split sequence $0 \rightarrow U \rightarrow V \rightarrow L \rightarrow 0$ in $\mathscr{C}$ and $X \in \mathscr{C}$ for $X$ is a direct summand of $V$. If $L$ is extension projective, it is projective and $X$ is not injective in $\bmod \Lambda$. Then there exists an almost split 
sequence $0 \rightarrow X \rightarrow L \oplus L^{\prime} \rightarrow Z \rightarrow 0$ in $\bmod \Lambda$ with $Z$ a direct summand of $M$, whence $Z \in \mathscr{C}$ and $X \in \mathscr{C}$ by (i).

From the preceding argument and its dual, we conclude that if $L \in$ ind $\mathscr{C}$ is not projective-injective in $\bmod \Lambda$, then for all irreducible maps $X \rightarrow L$ and $L \rightarrow Y$, we have $X, Y \in \mathscr{C}$. Let $\mathscr{D}=\left\{P_{i} \mid i=1, \ldots, s\right\}$ be the set of all those pairwise nonisomorphic projective-injective modules in ind $\Lambda$ for which the ends of the almost split sequence $0 \rightarrow \mathbf{r} P_{i} \rightarrow \mathbf{r} P_{i} / \operatorname{soc} P_{i} \oplus P_{i} \rightarrow P_{i} / \operatorname{soc} P_{i} \rightarrow 0$ [3, Proposition 4.11] are not in $\mathscr{C}$. Then $\mathscr{C}_{\mathscr{D}}$, the full subcategory of $\mathscr{C}$ determined by all modules having no direct summands in $\mathscr{D}$, has the following property. If $X \rightarrow Y$ is an irreducible map with $X, Y \in$ ind $\Lambda$, then $X \in \mathscr{C}_{\mathscr{D}}$ if and only if $Y \in \mathscr{C}_{\mathscr{D}}$.

Now suppose $\mathscr{C} \neq \bmod \Lambda$ is functorially finite. Then $\mathscr{C}_{\mathscr{D}}$ is functorially finite by [6, Propositon 3.13], and, according to [8, Corollary 2.2] and its dual, we have $\operatorname{Hom}_{\Lambda}(C, W)=0=\operatorname{Hom}_{\Lambda}(W, C)$ for all $C \in$ ind $\mathscr{C}_{\mathscr{D}}$ and $W \in($ ind $\Lambda)-\left(\right.$ ind $\left.\mathscr{C}_{\mathscr{D}}\right)$. Since $\Lambda$ is connected, indecomposable projective $\Lambda$ modules must either all be in ind $\mathscr{C}_{\mathscr{D}}$ or all belong to (ind $\left.\Lambda\right)-\left(\right.$ ind $\left.\mathscr{C}_{\mathscr{D}}\right) \neq \varnothing$. Clearly, the latter holds, i.e., ind $\mathscr{C}=\mathscr{D}$.

Remark 3. Proposition 2 is false without assumptions (ii) and (iii). Really, if $\Lambda$ is the group algebra of a finite group of order 2 over a field of characteristic 2 and $\mathscr{C}$ is the additive subcategory of $\bmod \Lambda$ generated by the trivial module, then $\mathscr{C}$ satisifies (i) and (iv) but is not closed under extensions.

Let $G$ be a finite group of order $|G|$ with a subgroup $H$ of index $[G: H]$. Let $k$ be a field of characteristic $p$ dividing $|G|$. From now on, put $\Lambda=$ $k G, \Gamma=k H$, and denote by $\mathscr{C}$ the full subcategory of relatively projective modules $[9,10]$ in $\bmod \Lambda$, i.e., of all modules isomorphic to a direct summand of $\Lambda \otimes_{\Gamma} X$ for some $X \in \bmod \Gamma$. It is well known that $\mathscr{C}$ is functorially finite, and the extension projective modules, as well as the extension injective modules in $\mathscr{C}$, are projective in mod $\Lambda$. In addition, $\mathscr{C}$ is closed under $D T r$ and $\operatorname{Tr} D$ by [4, p. 550]. Thus, Proposition 1 and 2 hold for every block of $\Lambda$. Since the necessary and sufficient conditions for the closure of $\mathscr{C}$ under extensions are well known, we can restate Proposition 2 as follows.

Corollary 4. $\mathscr{C}$ has almost split sequences if and only if $p$ is coprime to either $|H|$ or $[G: H]$.

Now suppose that $\mathscr{C}$ does not have almost split sequences. Since $\mathscr{C}$ is selfdual, it follows from Proposition 1 and from [7, Theorem 1.2], where the term extension projective should have been used instead of Ext-projective, that

(i) for some nonprojective $N \in$ ind $\mathscr{C}$, the middle term of the almost split sequence $0 \rightarrow L \rightarrow M \rightarrow N \rightarrow 0$ in $\bmod \Lambda$ is not in $\mathscr{C}$.

(ii) For some nonprojective $N^{\prime} \in$ ind $\mathscr{C}$, the kernel of one (and every!) right almost split morphism $V \rightarrow N^{\prime} \rightarrow 0$ in $\mathscr{C}$ is not in $\mathscr{C}$. Our next statement shows that for a given nonprojective module in ind $\mathscr{C}$, (i) and (ii) are equivalent.

Proposition 5. Let $0 \rightarrow L \stackrel{f}{\rightarrow} M \stackrel{g}{\rightarrow} N \rightarrow 0$ be an exact sequence in $\bmod \Lambda$ with $L \in \mathscr{C}$. Then

(a) In the exact sequence $0 \rightarrow K \rightarrow \Lambda \otimes_{\Gamma} M \stackrel{g m}{\rightarrow} N \rightarrow 0$, we have $K \simeq$ $L \oplus X(M)$, where $m: \Lambda \otimes_{\Gamma} M \rightarrow M$ is the multiplication map and $X(M)=\operatorname{Ker} m$. 
(b) $M \in \mathscr{C}$ if and only $K \in \mathscr{C}$.

(c) If $0 \rightarrow L \stackrel{f}{\rightarrow} M \stackrel{g}{\rightarrow} N \rightarrow 0$ is an almost split sequence in mod $\Lambda$, then $g m$ is a right almost split morphism in $\mathscr{C}$.

Proof. (a) We have the following exact commutative diagram of $\Lambda$-modules:

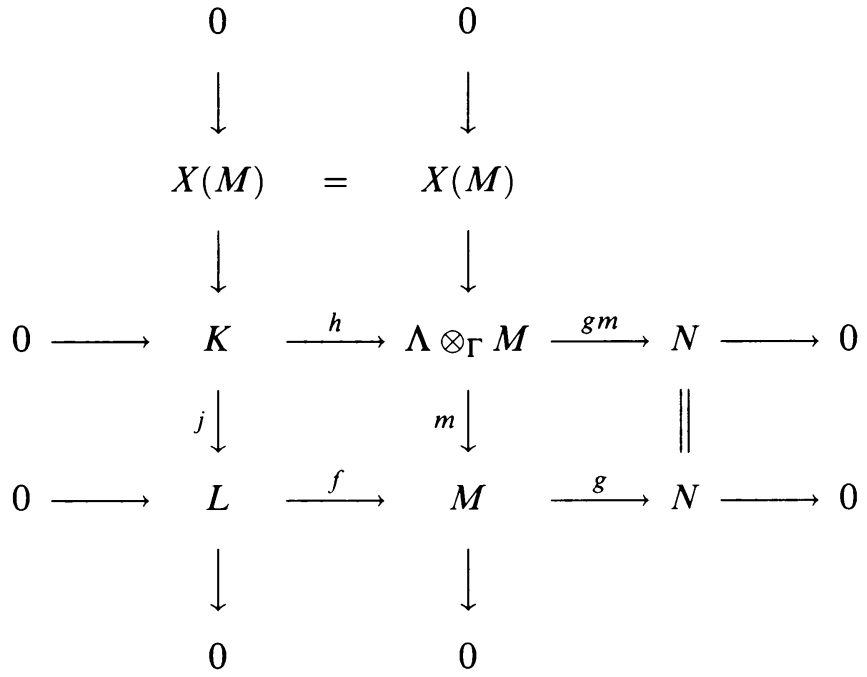

The middle column splits as a sequence of $\Gamma$-modules. Since $L$ is relatively projective, $f$ can be lifted to $\Lambda \otimes_{\Gamma} M$, and the left column splits.

(b) By the Krull-Schmidt theorem, it follows from (a) that $K \in \mathscr{C}$ if and only if $X(M) \in \mathscr{C}$, because $L \in \mathscr{C}$. Since every module in $\mathscr{C}$ is relatively injective and the middle column of the diagram splits over $\Gamma, X(M) \in \mathscr{C}$ if and only if $m$ is a splittable epimorphism over $\Lambda$, i.e., if and only if $M \in \mathscr{C}$.

(c) Clearly, $N \in \mathscr{C}$, and $g m$ is a right almost split morphism in $\mathscr{C}$ by the proof of [6, Proposition 3.10].

\section{ACKNOWLEDGMENT}

The results of the paper were obtained during the International Conference on Representations of Algebras at the University of Tsukuba in August 1990, where the author was fortunate to benefit from several long conversations with Idun Reiten. The author expresses his gratitude to Idun Reiten for her valuable advice and the time she so generously donated and to the organizers of the conference, in particular, to $\mathrm{H}$. Tachikawa for the opportunity to attend the conference. The author wishes to thank K. W. Roggenkamp for his statement and proof of Proposition 1 and Jon F. Carlson for several helpful suggestions.

\section{REFERENCES}

1. M. Auslander, Representation theory of Artin algebras. I, Comm. Algebra 1 (1974), 177-268.

2. M. Auslander and I. Reiten, Representation theory of Artin algebras. III. Almost split sequences, Comm. Algebra 3 (1975), 239-294.

3. - Representation theory of Artin algebras. IV. Invariants given by almost split sequences, Comm. Algebra 5 (1977), 443-518. 
4. __ Representation theory of Artin algebras. V. Methods for computing almost split sequences and irreducible morphisms, Comm. Algebra 5 (1977), 519-554.

5. M. Auslander and S. O. Smalø, Almost split sequences in subcategories, J. Algebra 69 (1981), 426-454.

6. __ Preprojective modules over artin algebras, J. Algebra 66 (1980), 61-122.

7. R. Bautista and M. Kleiner, Almost split sequences for relatively projective modules, J. Algebra 135 (1990), 19-56.

8. J. F. Carlson and D. Happel, Contravariantly finite subcategroies and irreducible maps, Proc. Amer. Math. Soc. (to appear).

9. G. Hochschild, Relative homological algebra, Trans. Amer. Math. Soc. 82 (1956), 246-269.

10. S. Mac Lane, Homology, Springer-Verlag, New York, 1967.

Department of Mathematics, Syracuse University, Syracuse, New York 13244-1150 